\title{
Abstracts from Texas Tech University Health Sciences Center School of Medicine Summer Research Program from the Lubbock Campus presented at the Student Research Week in March 2018
}

\author{
THE NAMES IN BOLD ARE THE MEDICAL STUDENTS WHO PARTICIPATED IN THIS PROGRAM IN 2017
}

THE AUTHOR INDEX STARTS AFTER THE LAST ABSTRACT (\#26)

\section{Impact of timing of interval cholecystectomy, following percutaneous cholecystostomy tube for acute cholecystitis, on operative and patient outcomes}

Fahad Ali, Eneko Larumbe, Edwin Onkendi

Background: Percutaneous cholecystostomy tube (PCT) has been used as a bridge treatment for grade II-III moderate to severe acute cholecystitis (AC) to "cool" the gallbladder down over several weeks and allow the inflammation to resolve prior to performing interval cholecystectomy (IC) and removal of the PCT. The aim of this study was to assess the impact of timing IC after PCT on operative success and outcomes.

Methods: A retrospective review of electronic medical records of patients who were treated for acute cholecystitis with a PCT and subsequently underwent IC at our institution between January 2005 to December 2016 was performed. The patients were divided into three groups $(n=7$ each) based on the duration of the PCT prior to IC, and these groups were comparatively analyzed. A comparative sub-analysis of clinical outcomes between patients who underwent surgery within the first week vs. third week or later after PCT was also performed.

Results: Overall, there was no statistically significant difference in outcomes between performing IC within the first 5 weeks, $5-8$ weeks and $>8$ weeks after PCT placement. The length of stay, overall morbidity, Clavien-Dindo grade of complications and mortality were similar between the 3 time intervals. However, a sub-analysis showed that patients who underwent IC within the first week of PCT placement had

DOI: $10.12746 /$ swrccc.v6i24.473 statistically significant higher mortality rate $(p=0.048)$ compared to those who underwent IC $>3$ weeks of PCT placement.

Conclusion: Delaying IC > 5 weeks after PCT placement for AC is not associated with any improvement in patient morbidity, length of stay or rate of conversion from laparoscopic to open cholecystectomy. Cholecystectomy within the first week of PCT placement is associated with higher mortality rate than after 3 weeks likely due to associated sepsis.

\section{Efficacy of Sm-p80 in natural mimic conditions in baboon analysis of IgM antibody titer and egg burden \\ Ryan Alonzo, Priscilla Ortiz, Jaxson Thomas, Whitni Redman, Arif Siddiqui, Samra Lazarus, Adebayo Molehin, Souad Sennoune, Weidong Zhang, A.A. Siddiqui}

Schistosomiasis is a tropical disease affecting a large number of populations. Five species of the Schistosoma genus parasite are associated with human disease. The species Schistosoma mansoni, was used for this study that mimics a natural chronic infection for intestinal schistosomiasis found in African countries. For this study, the vaccine contains Sm-p80 protein and a TLR9 agonist adjuvant, CpG-ODN. Sm-p80 is the large subunit of the parasite calpain and aids in surface membrane renewal a recycling. In this study, baboons were infected with $S$. mansoni cercariae, treated with praziquantel, immunized, and challenged with cercariae. Transformed pCold E. coli stock was used to express Sm-p80 protein to be used for ELISA antibody quantification. The protein was then purified and concentrated for ELISA antibody titers. Fecal egg counts were used throughout the study 
to demonstrate if the vaccine lowered egg burden. Liver, small intestine, and large intestine tissue egg counts were done to determine tissue egg load. Egg burden causes the majority of the pathology associated with the parasitic infection, so if the egg burden decreases there are less severe symptoms. The $\mathrm{Sm}-\mathrm{p} 80$ protein was accurately expressed, purified, and concentrated. IgM was focused on because not only is it the first antibody seen in an infection, but it would be helpful to determine if the vaccine would increase IgM titer levels. Although present, IgM titer levels did not show significant difference between control and experimental animals. Liver, small intestine, and large intestine tissue and fecal egg burdens were lowered in experimental animals post-vaccination.

\section{Impact of timing of interval cholecystectomy, following percutaneous cholecystostomy tube for acute cholecystitis, on operative and patient outcomes}

Usman Asad, Amir Aryaie, Eneko Larumbe, Mark Williams, Edwin Onkendi

Introduction: Percutaneous cholecystostomy tube (PCT) is used as a bridge treatment for grade II-III moderate to severe acute cholecystitis (AC) to allow the inflammation to resolve prior to performing interval cholecystectomy (IC) and removal of the PCT. The aim of this study was to assess the impact of timing IC after PCT on operative success and outcomes.

Methods/procedures: A retrospective review of EMRs of patients who were treated for AC with a PCT and subsequently underwent IC at UMC between January 2005 to December 2016 was performed. The patients were divided into 3 groups ( $n=7$ each) based on the duration of the PCT prior to IC and were comparatively analyzed. A comparative sub-analysis of clinical outcomes between patients who underwent surgery within the 1st week vs. 3rd week or later after PCT was also performed.

Results: Overall, there was no statistically significant difference in outcomes between performing IC within the first 5 weeks, $5-8$ weeks and $>8$ weeks after
PCT placement. The length of stay, overall morbidity, Clavien-Dindo grade of complications and mortality were similar between the 3 time intervals. However, a sub-analysis showed that patients who underwent IC within the first week of PCT placement had statistically significant higher mortality rate $(p=0.048)$ compared to those who underwent IC $>3$ weeks of PCT placement. The two patients who died in our sample had IC within a week after PCT placement. Even though there was a statistically significantly higher morbidity rate in those who had IC $>3$ weeks after PCT, the Clavien-Dindo grade of these complications was lower than III in all cases.

Conclusion: Delaying IC to $>5$ weeks after PCT placement for $A C$ is not associated with any improvement in patient morbidity, length of stay or rate of conversion from laparoscopic to open cholecystectomy. Cholecystectomy within the first week of PCT placement is associated with higher mortality rate than after 3 weeks likely due to associated sepsis.

\section{Accessing community connection to essential service survey (ACCESS survey)}

\section{Cheryl Erwin, Alyssa Byrd}

Background: Focal points for improving health policy revolve around minimizing deficiencies in access, quality, and cost of health care. Understanding the health care landscape of an area can aid in determining disparities in health care for underserved individuals. Additionally, individuals with genetic disorders are at risk for genetic discrimination, including access to care.

Objective: The goal of this study was to determine the rate of uninsured patients, assess perceptions of barriers to health care access, evaluate the importance of health care insurance, and inquire concerning patient perceptions of the risk to genetic privacy.

Methods: The study surveyed 23 adult patients in Lubbock, TX. The study used a 15-minute, anonymous, self-administered, pen and pencil survey (ACCESS Survey). It was distributed at TTUHSC clinics and assessed perceptions pertaining to access to health care and expectations of privacy of genetic information. 
Results: The uninsured and unemployment rates were reported to be higher than national average. Patients also had a lower average income than compared to the national average. The population worried about the cost of health insurance and out of pocket expenses more than access to care. Low numbers of patients had a personal care provider, but the wait time to see a family physician was less than the national average. The majority favored federal assistance for low-income persons and continued coverage with cost protection for individuals with pre-existing conditions. Patients worried more about genetic discrimination related to insurance over relationships and employment.

Conclusion: Health care in Lubbock, TX, is a major concern for patients. The cost of health insurance was especially worrisome to the population, though deficiencies in both access and quality exist in the area as well. These results show that work still needs to be done to decrease disparities in health care for the underserved population of rural west Texas.

\section{Chronic peritoneal indwelling catheters for the management of malignant and nonmalignant ascites: a narrative literature review}

Joseph Caldwell, Hawa Edriss, Kenneth Nugent

Background: Ascites is a debilitating condition affecting many patients with end-stage liver disease or advanced abdominal malignancies. Serial paracentesis can reduce the symptoms of refractory ascites, but this procedure requires frequent trips to a clinic and places a great burden on patients and their caregivers. Indwelling peritoneal catheters are an alternative which can allow these patients to manage their symptoms at home. This review aims to assess the safety and efficacy of these devices.

Methods: A literature search was conducted to identify articles reporting indwelling catheter placement in patients with ascites. Inclusion criteria were for studies with at least 20 adult subjects that had been published within the past 15 years. Patient demographics, indications, complication rates, and survival times were analyzed.
Results: Fourteen studies comprising 957 patients (687 with malignancy [71.7\%], 270 without [28.3\%]) were reviewed. Symptom improvement was reported in all cohorts. The most common complication in patients with malignant ascites was catheter dysfunction $(39 / 687,5.7 \%)$. Overall infection rate for patients with malignancy was $5.4 \%$ (37/687); patients with pancreatic malignancy comprised at least $70.2 \%(26 / 37)$ of these infections. The infection rate for patients with nonmalignant ascites was $12.2 \%$ (33/270), while catheter malfunction was $1.1 \%$ (3/270). Infection risks significantly increased for devices in place longer than 12 weeks. Average survival time after catheter placement was 7.2 weeks for patients with malignancy and 164 weeks for patients without malignancy.

Conclusions: Indwelling peritoneal catheters are effective for the palliation of refractory ascites in patients with certain malignancies. Due to prolonged device usage, peritonitis is a concern for patients with ascites attributable to nonmalignant etiologies, but proper implantation technique and maintenance may greatly reduce infection risks.

\section{Time to operation does not influence health outcomes for perforated peptic ulcer disease}

Esteban Esquivel, John Lung, Sharmila Dissanaike

Background: Perforated peptic ulcer (PPU) is usually considered a surgical emergency, with a mortality and morbidity up to $30 \%$ and $50 \%$, respectively. PPUs are associated with more than $70 \%$ of deaths in patients with peptic ulcer disease (PUD) and PPUs develop in $2-10 \%$ of patients with PUD. Prior studies in Europe and India have shown a link between rapid surgical intervention and positive outcomes in PPU.

Objective: We examined the relationship between the time interval from perforation to operation and postoperative outcomes.

Methods: Seventy-two patients were admitted to Texas Tech University Health Sciences Center in Lubbock, TX for a perforated peptic ulcer January 1 , 2010 - May 1, 2017. Data collected included the perforation to operation time interval, age, gender, ethnicity, income level (SES), insurance status, morbidity, 
mortality, and length of hospital stay. Logistic regressions were conducted using SPSS to determine predictive variables.

Results: Thirty-seven (51.4\%) patients had a perforation to operation time interval less than 24 hours, $14(19.4 \%) 24-48$ hours, and $21(29.2 \%)$ more than 48 hours. Thirty-three $(45.8 \%)$ patients had a postoperative morbidity, $20(27.8 \%)$ patients were readmitted, $9(12.5 \%)$ had postoperative mortality with an average time to death of 37.6 days, and $7(9.7 \%)$ had a postoperative surgical infection. Logistic regression analysis found no significant predictive variables for perforation to operation time interval, postoperative morbidity, or postoperative mortality.

Conclusion: Our results indicated that a longer perforation to operation time interval is not as consequential to patient outcome as suggested in previous studies outside the United States. Further research should be performed to examine whether this is due to advances in surgical and postoperative management in the United States that minimize the risk factor of a prolonged perforation to operation time interval.

\section{Serum therapy to prevent Pseudomonas aeruginosa sepsis: assessment of rPcrV-IgG in passively immunized mice}

Reed Farmer, Nithya Mudaliar, Jane ColmerHamood, Sharmila Dissanaike, John Griswold, Abdul Hamood

Pseudomonas aeruginosa is a gram-negative opportunistic pathogen that causes severe infections in immunocompromised patients including severely burned patients. Colonization of burnt tissue by $P$. aeruginosa often leads to systemic sepsis and death. Damage caused by $P$. aeruginosa is due to the production of numerous cell-associated and extracellular factors including the type three secretion system (T3SS) which translocates effector molecules into the cytoplasm of the host cell, resulting in cell death. In addition to the effector proteins, translocators form pores on the host cell membrane to facilitate effector translocation into the host cytosol.
Among these translocators is PcrV. We hypothesize that sufficient levels of PcrV-antibodies protect severely burned patients from $P$. aeruginosa sepsis. In this study, we tried to determine the level of PcrV antibodies within the blood of thermally injured mice following immunization with recombinant PcrV (rPcvR). We overproduced rPcrV in Escherichia coli and purified it using nickel column chromatography. Using rPcrV, we raised polyclonal PcrV antibody in rabbits. We purified the IgG fraction from rPcrV-immunized (rPcrV antibody [rPcrV-lgG]) and nonimmunized (control antibody [C-IgG]) rabbits using chromatography cartridges. Mice were intraperitoneally (IP) injected with either rPcrV-IgG or C-lgG at a dose of $14 \mathrm{mg} / \mathrm{kg}$. Blood was obtained from treated mice at $8,24,36,48,72$, and $96 \mathrm{~h}$ post-injection and the serum fraction was separated. The level of rPcrV-lgG in each serum fraction was determined by enzyme linked immunosorbent assay (ELISA). Between 8 and $72 \mathrm{~h}$ post injection, the titer of rPcrV-lgG was relatively constant (about 600-700 pmoles). At $96 \mathrm{~h}$ post injection, the titer dropped to 250 pmoles. These results suggest that thermally injured mice receiving a single IP injection of rPcrVIgG maintain a considerable titer that may protect them from $P$. aeruginosa sepsis for 4-5 days

\section{Isolated hip fracture mortality in geriatric trauma patients}

Tyson Fillmore, Kaushik Mukherjee, Jayne McCauley, Stephen Gates, Amber Tucker, Oscar D. Guillamondegui, Steven E. Brooks

Introduction: Geriatric isolated hip fracture patients have in-hospital mortality of 3\%, and one-year mortality between $20-33 \%$. Although studies detail frailty scoring and outcome prediction, few describe how to use this information. We recognized that some patients our Level 1 Trauma Center received early operative repair, only to transition to comfort care. We hypothesized that isolated hip fracture patients predominantly discharge to skilled nursing facilities (SNF) and have significant one-year mortality, justifying early consultation of Palliative/Supportive Care (PC) service for goals-of-care discussion. 
Methods: Retrospective cohort of 768 patients, aged 60 and older, was examined after treatment for isolated hip fracture between January 1, 2014 and December 31, 2016. Patients aged less than 60 years, pathologic fractures, and poly-system trauma patients were excluded. Data was analyzed using logistic regression for discharge disposition and mortality.

Results: Mean age was $79.5 \pm 9.2$ years. Mean length of stay was $6.1 \pm 3.2$ days. $3.4 \%$ of patients died in the hospital, $15.5 \%$ at 3 months, $17.9 \%$ at 6 months, and $25.6 \%$ at 1 year after injury. Only 34\% of patients went home; $14 \%$ to rehab, and $48 \%$ to SNF. Each decade of age increase, patients are 35\% less likely [95\% $\mathrm{Cl} 22-46 \% ; \mathrm{p}<0.001]$ to be discharged to home/rehab. Each decade of age increased odds of 6 -month mortality by $88 \%$ [ $95 \% \mathrm{Cl} 42-143 \%$; $p<0.001]$.

Conclusion: Only $34 \%$ of geriatric isolated hip fracture patients disposition home, and these fractures confer a $25 \%$ one-year mortality. This underscores the need for early consultation of PC for goals-of-care discussion with patients or surrogates.

\section{Analyzing anti-inflammatory effects of delta- tocotrienol on type ii diabetes}

Alan Gonzalez, Gurvinder Kaur, Michael Tomison, Kandis Wright, Chwan-Li Shen, Jannette M. Dufour

Type II Diabetes is a major epidemic affecting $9.3 \%$ of the US population. It is one of the leading causes of death in the US and is able to progress to other comorbidities that affects an individual's quality of life. For this study CD1 mice were fed a high fat diet to develop hyperglycemia and type II diabetes. Controls were fed a low fat diet and remained normoglycemic. Mice on the high fact diet were also treated with either: Delta-Tocotrienol, Statins, or a combination of two of these reagents. Since Delta-Tocotrienol is an anti-inflammatory member of the Vitamin $\mathrm{E}$ family, we hypothesized it would decrease the number of macrophages present in the pancreas, decrease insulin resistance and increase glucose clearance when compared to a high fat diet mouse. Insulin tolerance and glucose tolerance tests demonstrated that the Tocotrienol, Statin and combination groups had a decrease in insulin resistance and an increase in glucose clearance. Macrophages are leukocytes that can either be inflammatory or regulatory. They are thought to be inflammatory in these high fat models and may contribute to pancreatic islet beta cell damage. The number of macrophages in the pancreas were obtained by immunohistochemistry using paraffin embedded tissue sections and Galectin-3 antibody, a macrophage marker. Analysis of the results demonstrated that the Tocotrienol and combination groups had a decreased amount of macrophages compared to the high fat diet mouse, while the Statin group had a significant increase in macrophages. Overall this indicates that Delta-Tocotrienol could decrease inflammation and improve glucose homeostatsis in type II

\section{RLIP76 and GPx1 expression in adenocarci- noma and squamous cell carcinoma in lung tissue}

William C Green, Srikala Meda, Sharda Singh, Sanjay Awasthi

Rlip (aka RLIP76, encoded by RALBP1) and GPx1 (Glutathione peroxidase 1) are expressed in most human tissues. Rlip acts as a membrane-bound protein that transports xenobiotics and glutathione conjugates out of the cell. GPx1 functions in the detoxification of hydrogen peroxide and is an important antioxidant enzyme in humans, protecting normal cells from oxidative stress. Rlip is overexpressed in many cancers, while GPx1 is lost or under expressed. Increased Rlip expression contributes to increased drug resistance and loss of GPx1 contributes to cancer progression. Therefore, in this retrospective study, we investigated the prognostic value of Rlip and GPx1 expression in squamous cell and adenocarcinoma of the lung and compared their expression levels with surrounding normal tissue.

Tissue samples were taken from the South Plains Oncology Consortium for 19 cases of non-small cell lung cancer with 10 squamous cell and 9 adenocarcinomas. Formalin fixed and paraffin embedded tissues were sectioned into 4 micron thick slices. 
Tissues were prepared and stained with either antiRlip or anti-GPx1 primary antibodies and HRP secondary antibodies using the BenchMark ULTRA IHC/ ISH Staining Module by the TTUHSC Pathology Dept. Slides were interpreted and compared to negative controls by Dr. Suzanne Graham using light microscopy. Initial results indicated that Rlip stained more intensely in both squamous cell and adenocarcinoma tissue versus the surrounding normal tissue. GPx1 stained tissue was the opposite, in that both squamous cell and adenocarcinoma stained less than surrounding normal tissue. All these results indicate that less GPx1 expression may not influence the chemo/ radiotherapy response. On the other hand, protective effect of Rlip may be a basis for drug resistance during treatment and tumor growth. Further studies are in progress to confirm these findings and we will correlate expression of these proteins with chemo/radiotherapy response or survival.

\section{Dispersal of biofilms with commercially avail- able glycosidic hydrolases with potential appli- cations in wound healing}

Joel Barrett, Chia Hsu, Derek Fleming, Kendra Rumbaugh

Chronic wounds are often complicated by the presence of bacterial biofilms, which can confer up to one-thousand percent increase in antibiotic tolerance and maintain a persistent state of inflammation that makes wound-healing extremely difficult. Effective treatments must deal with this protective barrier, and the biofilm may be manually debrided or chemically disturbed to disperse the pathogens, exposing the bacteria to the host immune system and potential medical interventions. Previously, our lab has demonstrated degradation of polymicrobial biofilms with in vitro and ex vivo models using multiple glycoside hydrolases, enzymes that target common glycosidic linkages within bacterial biofilms. The present study investigated the effects of multiple enzymes on $S$. aureus and $P$. aeruginosa co-cultured biofilm materials within the in vitro and ex vivo models previously established by the Rumbaugh lab. An approximate $80 \%$ reduction in biomass produced by $P$. aeruginosa and $S$. aureus in vitro following exposure to either Xylanase or Cytohelicase $(p=0.05 ; n=3)$ was found. Furthermore, preliminary data obtained via q-PCR found approximately $45 \%$ and $6 \%$ dispersal within samples exposed to Cytohelicase and Xylanase respectively. Another goal of the study was to examine the effect of a particular combination of two common glycoside hydrolases (cellulase and alpha-amylase) on bacterial dispersal for a variety of bacterial species. Quantitative PCR data from these trials demonstrated high dispersal percentages for in vitro mono-bacterial samples. Together, these results suggest a potential dispersal and bactericidal mechanism for some of the glycosidic hydrolases analyzed in the present study. Future work will examine the efficacy of these enzymes in vivo, as well as explore a wider variety of enzyme combinations, in order to estimate the clinical relevance of commercially available glycosidic hydrolases in treating biofilm-containing wounds.

\section{Student knowledge integration of life lessons in spirituality}

\section{Cheryl Erwin, Adam Judd}

Background: Religion and spirituality are important to many patients; and connecting with patients on a spiritual level can benefit patient care. These types of connections can help build patient trust, increase satisfaction with care, and enhance patient intention to adhere to physician recommendations (Street, 2008). However, lack of training, concerns about appropriateness, and questions about patient interest can all keep physicians from inquiring about patients' spirituality (Baetz, 2004; Rasinski, 2011). This disconnect is undermining the patient-physician relationship, and many patients are demanding that their physicians be able to address religiosity and spirituality with them (Kuczewski, 2007).

Purpose: This study aims to evaluate the perspective of students and physicians at Texas Tech University Health Sciences Center regarding curriculum in the spiritual dimension to health care in general and to determine their preference, in particular, with regards to inclusion of spirituality in their training.

Procedures: A survey was distributed among current students and faculty at TTUHSC. Responses 
of the faculty and students were compared. Of the responses that were statistically distinct, we did a thematic analysis and allocated the responses into four groups: diversity, communication, end of life needs, and understanding of resources available. Free-text responses about the pros and cons of addressing spirituality in healthcare written by the students and faculty were also qualitatively analyzed.

Conclusions: Many students are uncomfortable addressing spirituality in a healthcare setting, and further education on the topic is warranted.

\section{Prophylactic antibiotic usage is not associated with any difference in postoperative UTI-related complications after ileal conduit urinary diversion}

\section{Carson Kirkpatrick, Allen Medway, Pranav Sharma}

Introduction: Majority of complications after ileal conduit urinary diversion with cystectomy are related to urinary tract infections (UTIs). Controversy exists regarding use of prophylactic antibiotics after surgery. We determined if prophylactic antibiotic use during ureteral stent placement after ileal conduit urinary diversion decreased incidence of UTI-related complications.

Methods: We retrospectively identified 75 consecutive patients who underwent ileal conduit urinary diversion with cystectomy at our institution from 2010-2016. Patients were stratified based on presence or absence of a UTI-related complication in the 90-day postoperative period. Means were compared with independent T-test and proportions with chisquare analysis. Multivariate logistic regression was performed to determine independent predictors of UTI-related complications.

Results: Forty-five patients (60\%) were prescribed prophylactic antibiotics after surgery. Mean duration of antibiotic use was 15 days, and mean duration of ureteral stenting was 25 days. Most common antibiotics used included fluoroquinolones ( $n=23,30.7 \%)$ followed by sulfamethoxazole-trimethoprim $(n=14$, $18.7 \%$ ). Rate of 90 -day UTI-related complications was $36 \%(n=27)$, and 90 - day UTI-related readmission rate was $14.7 \%(n=11)$. On bivariate and multivariate analysis, prophylactic antibiotic use was not associated with reduced 90-day UTI-related complications $(p>0.05)$. Patients prescribed prophylactic antibiotics had increased incidence of Clostridium difficile infections in the 90-day postoperative period compared to controls ( $20 \%$ vs $3.3 \%$; $p=0.038$ ).

Conclusions: Prophylactic antibiotic use after ileal conduit urinary diversion with cystectomy was not associated with reduced UTI- related complications, and rate of Clostridium difficile infections was higher in this patient cohort. The effect of early removal of ureteral stents on UTI risk still has to be elucidated.

\section{Mortality and pneumonia rates in rib fractures: a National Trauma data bank review 2010-2014}

Yana Puckett, Lydia Kong; Hannah Pham; Sharmila Dissanaike, Steven E. Brooks

Objectives: Surgical stabilization of rib fractures, aggressive pulmonary toilet, and epidural and paravertebral blocks have become mainstay treatment and increasingly popular in the management of trauma patients with rib fractures. Previous studies have associated increased age and number of rib fractures with increased mortality and complication prompting a paradigm shift in treatment. We hypothesize that mortality and pneumonia rates have decreased over the years due to improvements in rib fracture management.

Methods: Retrospective NTDB data was extracted between 2010-2014 for patients with rib fractures (dcodes 807-807.4). Patient demographics, number of fractured ribs, and pneumonia and mortality rates were abstracted. Patients were dichotomized by age $<65$ or $\geq 65$. Pearson's correlation was used to compare trends in mortality and pneumonia. Chi-Square test was used to compare mortality and pneumonia rates with levels of rib fractures. Significance at $\leq 0.05$.

Results: A total of 789,769 rib fracture patients were analyzed. Total overall mortality and pneumonia rates were $0.29 \%$ and $9.01 \%$, respectively. In patients $\geq 65$, overall mortality rate decreased by $0.14 \%$ $(\mathrm{R} 2=-0.623, \mathrm{P}=0.262)$ and pneumonia rate decreased by $1.55 \%(R 2=-0.986, P=0.002)$. In patients $<65$, 
there was no significant change in overall mortality $(\mathrm{R} 2=-0.189, \mathrm{P}=0.761)$ or overall pneumonia $(\mathrm{R} 2=-0.283, \mathrm{P}=0.644)$ rates. There was no statistical difference in either mortality or pneumonia rates in conjunction to rising number of rib fractures in either age group.

Conclusions: Improved management and treatment of rib fractures over time (2010-2014) has contributed to the observed decrease of overall rates in both mortality and pneumonia in patients $>65$ with rib fractures. Rising number of rib fractures may no longer be associated with concomitant increase in pneumonia and mortality rates.

\section{Developing a database for forensic analysis: impact of water temperature and exposure time on scald burns in human skin}

\section{Audrey Le, Natalie Tully, Sharmila Dissanaike}

Introduction: Determining the time of exposure to a given water temperature is a valuable tool in assessing the etiology of a scald. This becomes extremely important in forensic analysis in the setting of suspected child and elder abuse. It is known that increasing the water temperature not only decreased the time to scald, but also increases the severity of the scald; however, little research has been done to accurately predict these measures on fresh human skin. Furthermore, the available data does not account for variations in age and ethnicity. Considering what these determinations may expose about the nature of the patient's condition, we sought to increase the accuracy of available data.

Methods: Patients undergoing elective surgery of healthy tissue from the abdomen and lower limb donated the removed tissue for this study. Immediately after surgery, the skin was dissected into $2 \mathrm{~cm} \times 2 \mathrm{~cm}$ samples and exposed to water baths starting at 50 degrees and visualizing the time it took to develop a second degree and third degree scald. The skin was then discarded and the trial was repeated with a fresh sample from the same tissue at 60, 70, 80 and 90 degrees.

Results: In this study, skin was obtained from 11 patients of Caucasian and Hispanic descent. Time to second and third degree scald decreased rapidly as water temperature increased. Differences in time to burn were noted at lower temperatures, and variability among patients decreased as the temperature increased.

Conclusions: There is variability in time to scald in human skin at lower temperatures, which narrows with increasing water temperature. We are expanding this study to a larger sample size in order to build a robust reference tool. The results of this study will provide the groundwork for more reliable estimation of the time and temperature necessary to cause a scald burn. This will improve our ability to provide determinations of non-accidental injury and direct preventive measures.

\section{The association between body mass index and airway pressures in patients with sepsis and acute respiratory failure}

Michelle Lear, Hawa Edriss, Asley Sanchez, Edna Juarez, Shengping Yang, Kenneth Nugent

Purpose: Patients with increased BMI have excessive adipose tissue in the thoracic wall and abdomen. This reduces chest wall compliance and creates worse gas exchange secondary to abnormal ventilation/perfusion relationships in the lung bases. This study considers the effect of obesity on the pressures required for mechanical ventilation in patients with sepsis and acute respiratory failure.

Methods: The EMR of patients hospitalized between 2010 and 2016 with sepsis who required mechanical ventilation were reviewed to collect demographic characteristics, clinical information including $\mathrm{BMI}$, mechanical ventilation pressures, management requirements, and outcomes including mortality and length of stay in the ICU and in the hospital. Peak pressures and plateau pressures were recorded 24 hours after admission and the initiation of mechanical ventilation. This timeframe allowed clinicians to adjust the ventilator and stabilize the patient.

Summary: This study included 173 adult patients. The mean age was $58.5 \pm 16.7$ years; $53.2 \%$ were men. The mean BMI was $29.6 \pm 11.9$. The mean 
white blood count was $14.3 \pm 8.0 \mathrm{k} / \mu \mathrm{L}, 43.9 \%$ of the patients had pulmonary infections, and $34.7 \%$ had extrapulmonary infections. The overall mortality was $44.5 \%$. The mean length of stay was $12.4 \pm 11.8$ days in the ICU and $16.6 \pm 13.6$ days in the hospital. The mean peak pressure on day one of mechanical ventilation increased from $19.5 \pm 5.1 \mathrm{~cm} \mathrm{H}_{2} \mathrm{O}$ in underweight patients (BMI <18.0) to $26.0 \pm 8.0 \mathrm{~cm} \mathrm{H}_{2} \mathrm{O}$ in patients in the obese category $(\mathrm{BMI}>30)$. The mean plateau pressure on day 1 of mechanical ventilation increased from $16.3 \pm 4.3 \mathrm{~cm} \mathrm{H}_{2} \mathrm{O}$ in underweight patients to $21.3 \pm 5.5 \mathrm{~cm} \mathrm{H}_{2} \mathrm{O}$ in obese patients.

Conclusions: These results indicate that patients with increased BMI require higher average ventilator pressures to maintain adequate gas exchange. This likely reflects reduced chest wall compliance and suggests that trans-pulmonary pressures are less certain in these patients.

\section{Long-term growth, neurodevelopmental, and systemic outcomes in laser and bevacizumab- treated infants with retinopathy of prematurity}

Margaret Littlejohn, Lingkun Kong, Ann Demny, Robert G. Voigt, Sonia A. Monteiro

Purpose: Recent usage of intravitreal bevacizumab (IVB) to treat retinopathy of prematurity (ROP) has led to questions regarding systemic absorption of bevacizumab and its potential long-term side effects. We performed a prospective, observational clinical study to test the hypothesis that IVB-treated infants have similar long-term growth and systemic outcomes compared to laser-treated infants.

Methods: Total of 67 infants who were treated with IVB injection ( $N=47)$ or laser $(N=20)$ from 2010 to 2014 were enrolled. The neurodevelopmental outcome measurements include body weight (BW), height and head circumference (HC) at age 1 and 3; neurodevelopmental quotient (DQ) at age 1 and 3. Systemic multi-organ functional outcomes include hepatic, renal, and hematologic lab values. Two-tailed Student's t- tests were used to compare the group means of the IVB-treated and laser-treated groups.
Results: There was global developmental delay in both groups. Patients in both groups showed progress over time, but the changes were not significant, $p=0.1$ to 0.7 . There were no significant differences in neurodevelopmental sub-domains, body weight, height and $\mathrm{HC}$ between the two groups at age 1 and $3, p=0.3$. Infants treated with IVB had lower AST levels $(p=.002)$ at 2 months post treatment as well as lower albumin levels $(p=.034)$ at 4 weeks post treatment. IVB treated infants showed lower creatinine levels $(p=.013)$ at 2 months post treatment. Other renal lab values showed non-significant differences in groups. IVB treated infants demonstrated significantly lower blood glucose $(p=.041)$ at 2 years of age. No significant differences between IVB and laser treated infants were demonstrated in hemoglobin, hematocrit, or platelet counts.

Conclusions: Our results indicate that there are differences in liver, kidney and blood glucose lab tests between IVB and laser treated infants. The clinical significance of these changes needs to be investigated.

\section{Moderate or severe LUTS is associated with increased recurrence of non-muscle-invasive urothelial carcinoma of the bladder}

\section{Austin Lunney, Pranav Sharma MD, Allan Haynes}

Introduction: Non-muscle-invasive bladder cancer can recur despite transurethral resection (TURBT) and adjuvant intravesical therapy. Tobacco products excreted in urine are hypothesized to cause tumor-promoting effects on urothelial cells through direct contact via mechanisms such as immunomodulation. We determined if moderate or severe lower urinary tract symptoms (LUTS) (defined as International Prostate Symptom Score [IPSS] >8) was associated with increased tumor recurrence.

Methods: We retrospectively identified 70 consecutive men initially diagnosed with non-muscle-invasive urothelial carcinoma of the bladder at our institution from 2010 to 2016. Patients were stratified based on presence or absence of tumor recurrence on follow-up. Means were compared with independent T-test and proportions with chisquare analysis. Multivariate logistic regression 
was performed to determine independent predictors of recurrence.

Results: Majority of patients had Ta disease $(58.6 \%)$ followed by T1 (28.6\%) and Tis (12.9\%). Forty-one $(58.6 \%)$ patients had moderate or severe LUTS upon presentation within 30 days of initial TURBT with mean IPSS of 13.2 vs 5.2 in control group ( $p<0.01)$. Biopsy-proven tumor recurrence occurred in 24 (34.3\%) patients at mean follow-up of 31.7 months. Mean time to recurrence was 14.6 months. Twenty-two of $41(53.7 \%)$ patients with moderate or severe LUTS developed tumor recurrence vs 2 of $29(6.9 \%)$ controls $(p<0.01)$. Moderate or severe LUTS was an independent predictor of tumor recurrence (odds ratio [OR]: 20.7, 95\% confidence interval [Cl]: $3.3-131 ; p=0.001)$.

Conclusions: Contact time with urine may be an important prognostic factor in non-muscleinvasive bladder cancer. Patients with significant urinary symptoms should be treated aggressively to minimize recurrence risk.

\section{Incidental radiological finding leading to neo- natal Herpes Simplex virus diagnosis}

Mary Miller, Fatma Levent, Roy Jacob

Neonatal Herpes Simplex Virus (HSV) can result in permanent sequelae despite its low prevalence. Central nervous system (CNS) involvement occurs in approximately one-third of the cases, with typical manifestations including seizures, lethargy, poor feeding, and skin lesions. In the absence of skin lesions, the initial presentation of HSV CNS disease may be indistinguishable from other causes of neonatal sepsis or meningitis.

We report a case of a two-week old female born at 32 weeks of gestation presenting with intrauterine growth retardation (IUGR) and respiratory distress. The mother denied any history of cold sores, genital ulcers, or vesicles. The patient was started on empiric antibiotics which were discontinued since the cultures remained negative. She had a normal physical examination, however continued to have poor feeding. On day of life 10, a head echoencephalogram
(US) was significant for mild dilation of ventricles with increased echogenicity. A repeat Head US was consistent with ventriculitis which lead to a Magnetic Resonance Imaging revealing diffuse leptomeningeal and periventricular enhancement. A lumbar puncture was performed; cerebrospinal fluid showed pleocytosis with lymphocytic predominance. Gram stain and culture remained negative, but an HSV-1 polymerase chain reaction (PCR) was positive. The patient initially received empiric acyclovir, and antibiotics which were discontinued once cultures were negative. She continued treatment with intravenous (IV) acyclovir for 21 days. She was discharged to continue oral acyclovir until six months of age. Although neonatal HSV infection with CNS involvement commonly presents with seizures, lethargy, irritability or poor feeding, early in the course, none of these symptoms may be present. Evaluation for HSV infection and empiric acyclovir treatment should be considered in all neonates with aseptic meningitis or other signs or symptoms of meningoencephalitis without an obvious bacterial cause.

\section{Efficacy of Sm-p80 in natural mimic condi- tions in baboon: determination of IgG1 antibody titer and egg burden}

\section{Priscilla Ortiz, Ryan Alonzo, Jaxson Thomas, Whitni Redman, Arif Siddiqui, Samra Lazarus, Adebayo Molehin, Souad Sennoune, Weidong Zhang, A.A. Siddiqui}

Schistosomiasis is a devastating parasitic neglected tropical disease that currently affects hundreds of millions of individuals. Due to the complex life cycle of schistosomes, inefficient control measures currently in place, and risk of drug resistance to praziquantel (PZQ), an alternative elimination strategy is imperative. While several vaccines for schistosomiasis are in various stages of animal and human trials, the Sm-p80 vaccine shows significant promise. Targeting the membrane renewal protein calpain, the Sm-p80 vaccine is the only schistosomiasis vaccine shown to have prophylactic as well as therapeutic effects. In acute murine and baboon studies, Sm-p80+CpG-ODN vaccine had the ability to increase total IgG titers, and decrease the amount 
of S. mansoni eggs passed in stool and retained in tissues. Any proposed schistosomiasis vaccine will primarily target populations in endemic regions. As individuals in these regions have likely been infected with schistosomiasis and given $\mathrm{PZQ}$ treatment at a previous point, it is important to consider the effects of past exposures and treatment to schistosomiasis when testing a vaccine. In this study, 10 baboons were chronically infected with Schistosoma mansoni and received $P Z Q$ treatment to mimic natural infection conditions in endemic communities. Baboons were divided into two groups $(n=5)$, with the experimental group receiving the Sm-p80 + CpG-ODN vaccine and the control group receiving CpG-ODN vaccine both followed by two boosters. The experimental group expelled fewer eggs in their stool when compared to the control group. Experimental baboons also exhibited less egg retention in the liver (36.7\%), small intestine (74.9\%), and large intestine (51.7\%) compared to control baboons. An increased IgG1 titer was also detected during post-vaccination cercarial challenge with $\mathrm{S}$. mansoni in experimental baboons in comparison to control baboons, which suggests an increased protection against $S$. mansoni in experimental baboons.

\section{Possible role of RALBP1 in regulating AP2M1 effects on endocytosis, immune response and blood sugar regulation}

\section{Aditya Rajan, Sanjay Awasthi, Sharda Singh}

RALBP1, a mercapturic acid pathway, endocytosis related gene, has attracted interest due to its role in the pathogenesis of various cancers. Studies have shown RALBP1 deficient mice have increased p53 activation, decreased glucose and lipid levels, and a high degree of resistance to carcinogenesis even in the presence of potent carcinogens. Studies have shown RALBP1 binds to AP2M1, and AP2M1 is an inhibitor of the insulin secretion promoting protein GLP-1. we sought to outline the possible effects of this interaction on regulation of endocytosis, Carcinogenesis and Insulin secretion. We surveyed RALBP1 binding information from the database Uniprot and found AP2M1 to be a gene of particular interest. We used RNA sequencing data from p53 -/- mice that had methylated RALBP1 promoter regions to analyze AP2M1 and GLP1 expression levels. We used the bioinformatics database Cbioportal to reveal the relationship between RALBP1 and AP2M1 gene expression levels. We reviewed articles on the database Genecards that outlined the pathways AP2M1 participates in and its effects on endocytosis, immune response to cancer and blood sugar regulation. Data from RNA Sequencing revealed that AP2M1 was up-regulated in p53 -l- mice upon RALBP1 knockdown. AP2M1 binds CTLA-4 at residues 152-174 and reduces cell surface expression of CTLA-4 through endocytosis. AP2M1 binds to GLP-1 reducing cAMP concentrations from $2.3 \mathrm{pmol} / \mathrm{L}$ to $1.7 \mathrm{pmol} / \mathrm{L}$. Knockdown of AP2M1 results in GLP-1 induced insulin secretion increasing by $50 \%$. AP2M1 may reduce Tumor expansion by inhibiting CTLA-4. It may also play a role in increasing insulin receptor sensitivity in RALBP1 knockout mice by decreasing GLP-1 induced insulin secretion. Based on the downstream effects of AP2M1 and the known effects of RALBP1 on these pathways, we hypothesize that RALBP1 down-regulates AP2M1 expression and up-regulates GLP-1 expression. It follows that RALBP1 may inhibit the immune response to cancer and play a role in Type II Diabetes.

\section{Lubbock centenarians' beliefs on fac- tors affecting longevity: A pilot study through interview}

Lisa Saa, Catherine Hudson, Gordon Gong, Billy U. Phillips J

According to the US 2010 census, the number of centenarians has increased by $5.8 \%$ from 2000 , with 7 male and 28 female centenarians per 100,000 population. Prior literature has explored individual behavior determinants of health, finding a wide variety of responses, as well as biological and genetic social determinants of health. However, studies comparing factors affecting longevity in urban versus rural centenarians have not been conducted. The purpose of this study is to determine centenarians' self-perception on longevity.

Inclusion criteria required individuals to be a Lubbock county resident, aged $>90$ years old, and 
without dementia. Participants were recruited through word of mouth and local newspaper advertisement. Researchers met with participants in their homes to conduct interviews which consisted of 14 direct questions, and one open ended question. Topics included: most recent residences, earliest memories, most memorable events, hobbies, favorite jobs, lifetime heroes, places where they have been happiest, modern conveniences, attitude changes, physician access, self-perception of longevity, advice to a 20-year-old, and anything left they would like to accomplish. Five males and five females were interviewed, with an average age of 92.4 years. The cohort lived in Lubbock County an average of 48.4 years. Earliest memories included living on a farm (3/10) and family-centered events (3/10). Their most memorable events included serving in or working with the military during WWII (5/10). When asked who were their lifetime heroes, $4 / 10$ stated no one. They were happiest in places where they were with family or surrounded by community (8/10). Lastly, many participants stated they either had nothing left they wanted to accomplish or that they just wanted to live out their days well.

Most Lubbock centenarians believe a combination of good genes, diet and exercise, and productive work to do throughout life are contributing factors to their longevity.

\section{The association between body mass index and gas exchange in patients with sepsis and acute respiratory failure}

Asley Sanchez, Hawa Edriss, Edna Juarez, Michelle Lear, Shengping Yang, Kenneth Nugent

Obese patients with reduced chest wall compliance usually have reduced trans-pulmonary pressures, especially at the lung bases, during mechanical ventilation. This likely reduces regional lung volumes during mechanical ventilation and creates more abnormal ventilation/perfusion relationships. This study considers the effect of body mass index (BMI) on gas exchange measured by $\mathrm{PaO}_{2} / \mathrm{FiO}_{2}$ ratios and required PEEP levels during sepsis. The electronic medical records of patients hospitalized between 2010 and 2016 with sepsis who required mechanical ventilation were reviewed to collect demographic characteristics, clinical information including BMI, pressures required for mechanical ventilation, management requirements, and outcomes including mortality and length of stay in the ICU and in the hospital. PEEP pressures and $\mathrm{PaO}_{2} / \mathrm{FiO}_{2}$ were recorded 24 hours after admission to the medical intensive care unit and the initiation of mechanical ventilation. This timeframe allowed clinicians to adjust the ventilator and to stabilize the patient.

This study included 173 adult patients. The mean age was $58.5 \pm 16.7$ years; $53.2 \%$ were men. The mean BMI was $29.6 \pm 11.9$. The mean white blood count was $14.3 \pm 8.0 \mathrm{k} / \mu \mathrm{L}, 43.9 \%$ of the patients had pulmonary infections, and $34.7 \%$ had extrapulmonary infections. The overall mortality was $44.5 \%$. The mean length of stay was $12.4 \pm 11.8$ days in the ICU and $16.6 \pm 13.6$ days in the hospital. The mean $\mathrm{PaO}_{2} /$ $\mathrm{FiO}_{2}$ ratio decreased from $251 \pm 14$ in the underweight patients to $185 \pm 11$ in the obese patients. The mean PEEP level increased from $5.6 \pm 1.3 \mathrm{~cm} \mathrm{H}_{2} \mathrm{O}$ in the underweight patients to $6.4 \pm 2.6 \mathrm{~cm} \mathrm{H}_{2} \mathrm{O}$ in the obese patients. These trends in $\mathrm{PaO}_{2} / \mathrm{FiO}_{2}$ ratios PEEP levels across BMI categories were not statistically significant. These results suggest that gas exchange based on $\mathrm{PaO}_{2} / \mathrm{FiO}_{2}$ ratios is worse in obese patients with acute respiratory failure associated with sepsis, but these differences did not reach statistical significance.

\section{Efficacy of Sm-p80 in natural mimic condition in baboon: analysis of IgA antibody titer and egg burden}

\section{Jaxson Thomas, Ryan Alonzo, Priscilla Ortiz,} Whitni Redman, Arif Siddiqui, Samra Lazarus, Adebayo Molehin, Souad Sennoune, Weidong Zhang, A.A. Siddiqui

Schistosomiasis is a neglected tropical disease with an estimated 400-600 million people currently infected. Despite control measures, such as praziquantel $(P Z Q)$ treatment and snail eradication, the disease continues to spread to new areas. The discovery of a protective vaccine remains the most potentially effective means for disease control. The goal of a vaccine is to reduce the morbidity of schistosomiasis 
by lowering the parasitic load, hindering egg production and protecting against acute and chronic schistosomiasis. Previous work has shown that a vaccine based upon Schistosoma tegument protein Sm-p80 with adjuvant to elicit an effective immune response. In endemic areas, people with schistosomiasis are treated with $P Z Q$ which does not protect against reinfection. This study seeks to explore whether the vaccine provides greater protection than PZQ treatment alone as a more effective control method. In order to mimic natural conditions in which this vaccine would be given, baboons have been challenged with a Schistosoma mansoni infection and then treated with $P Z Q$. Experimental baboons were immunized with Sm-p80 + ODN and re-challenged with Schistosoma. At various time points fecal and serum samples were collected. At the end of the experiment organ, tissue and blood samples were collected. Results were obtained after counting eggs in liver, small intestine and large intestine samples. Fecal samples from 6 time points along the experiment were counted. ELISA tests were also performed for each baboon sera from 5 time points for IgA against Sm-p80. The vaccine was found to have a protective effect. We found that the amount of eggs in tissue samples was significantly reduced. The liver burden decreased by $32.6 \%$, the small intestine by $53.8 \%$ and the large intestine by $49.6 \%$. Fecal counts revealed that vaccinated baboons exhibited a reduced egg burden compared to non-vaccinated baboons. IgA titers showed responses to sm-P80 in both experimental and control animals.

\section{The synthetic retinoid fenretinide induces MYCN downregulation in neuroblastoma cell lines}

Eduardo Urias, Thinh Nguyen, Balakrishna Koneru, Sun J. Wei, Min H. Kang, C. Patrick Reynolds

Use of 13-cis retinoic acid (13-cisRA) as part of maintenance-phase chemotherapy of high-risk neuroblastoma significantly improves outcome, prompting investigation of other retinoids with anti-cancer properties for treating neuroblastoma. Fenretinide is a synthetic retinoid currently in clinical trials for treatment of several types of cancer including neuroblastoma. Fenretinide acts in a p53-independent manner to induce apoptosis, by increasing intracellular levels of reactive oxygen species and dihydroceramides. MYCN genomic amplification is an oncogenic driver of many high-risk neuroblastomas and high MYCNexpressing neuroblastomas have been shown to be particularly sensitive to fenretinide. In the current study we determined the effect of fenretinide on MYCN expression in patient-derived neuroblastoma cell lines. MYCN expression was found to be downregulated both at the protein and mRNA level as early as 12 hours after exposure to clinically-achievable concentrations of fenretnide. These data point toward a mechanism by which fenretinide can contribute to tumor cell cytotoxicity in neuroblastoma by inducing a downregulation of this important oncogene.

\section{Gender/ethnic differences in seeking health- care plus time of recovery from procedures for shoulder/knee conditions}

Mimi Zumwalt, George Brindley, Ali Ashraf, Adam Woolridge, Rhett Butler, Amanda Weaver, John Chapa, Anudeep Dasaraju

Previous papers in North America have demonstrated that females exhibit more severe pain in more locations on the body than males, but tend to wait longer before seeking help for musculoskeletal issues. Also, an unconscious bias exists within physicians/ surgeons in terms of recommending specialist referral and/or surgery for males over female patients. Finally, initial orthopedic presentation of joint pain in females usually is more severe which impacts the postoperative outcome as outcomes tend to be less successful for females.

This work is licensed under a Creative Commons Attribution-ShareAlike 4.0 International License. 


\section{ApPendiX}

\begin{tabular}{|c|c|c|}
\hline Last Name & First Name & Abstract Number \\
\hline Ali & Fahad & 1 \\
\hline Alonzo & Ryan & $2,20,24$ \\
\hline Asad & Usman & 3 \\
\hline Barrett & Joel & 11 \\
\hline Byrd & Alyssa & 4 \\
\hline Caldwell & Joseph & 5 \\
\hline Esquivel & Esteban & 6 \\
\hline Farmer & Reed & 7 \\
\hline Filmore & Tyson & 8 \\
\hline Gonzales & Alan & 9 \\
\hline Green & William & 10 \\
\hline Hsu & Chia & 11 \\
\hline Judd & Adam & 12 \\
\hline Kirkpatrick & Carson & 13 \\
\hline Kong & Lydia & 14 \\
\hline $\mathrm{Le}$ & Audrey & 15 \\
\hline Lear & Michelle & 16,23 \\
\hline Littlejohn & Margaret & 17 \\
\hline Luney & Austin & 18 \\
\hline Miller & Mary & 19 \\
\hline Ortiz & Priscilla & $2,20,24$ \\
\hline Rajan & Aditya & 21 \\
\hline Saa & Lisa & 22 \\
\hline Sanchez & Asley & 16,23 \\
\hline Thomas & Jaxson & $2,20,24$ \\
\hline Urias & Eduardo & 25 \\
\hline Weaver & Amanda & 26 \\
\hline
\end{tabular}

